\title{
A Case of Sustained Objective Response of Recurrent/Progressive Diffuse Intrinsic Pontine Glioma with Phenylbutyrate and Targeted Agents
}

\author{
Stanislaw R. Burzynski, Gregory S. Burzynski, Sheldon Brookman \\ Burzynski Clinic, Houston, USA \\ Email: srb@burzynskiclinic.com \\ Received 7 December 2014; revised 28 December 2014; accepted 6 January 2015 \\ Academic Editor: Amit Kumar Tyagi, The University of Texas MD Anderson Cancer Center, USA \\ Copyright (C) 2015 by authors and Scientific Research Publishing Inc. \\ This work is licensed under the Creative Commons Attribution International License (CC BY). \\ http://creativecommons.org/licenses/by/4.0/ \\ (c) (i) Open Access
}

\begin{abstract}
Diffuse intrinsic pontine glioma (DIPG) is the most common type of brainstem glioma and one of the most deadly brain tumors. DIPG in young adult patients is a rare disease for which treatment options are limited. Radiation therapy remains the standard-of-care for newly-diagnosed DIPG, but no established therapies for recurrent disease are available. This paper describes the results of treatment of a young adult patient diagnosed with DIPG that progressed after radiation therapy. Therapy included sodium phenylbutyrate (PB) in combination with the targeted agents: pazopanib, everolimus, erlotinib, and bevacizumab. The patient achieved a rapid partial response, which persisted over a year and five months. The patient opted to discontinue the therapy and thereafter elected chemotherapy, which resulted in a subsequent rapid progression and death within one month. The targeted treatment was associated with minor toxicity that included a Grade 2 skin rash and Grade 1 elevation of transaminases. In conclusion, a combination of PB and currently available targeted drugs may offer extended survival in patients with recurrent DIPG.
\end{abstract}

\section{Keywords}

Antineoplastons, Brainstem Glioma, DIPG, Phenylbutyrate, Targeted Agents, Treatment of DIPG

\section{Introduction}

Diffuse intrinsic pontine gliomas (DIPG), the most common type of brainstem glioma, are uniformly deadly tu-

How to cite this paper: Burzynski, S.R., Burzynski, G.S. and Brookman, S. (2015) A Case of Sustained Objective Response of Recurrent/Progressive Diffuse Intrinsic Pontine Glioma with Phenylbutyrate and Targeted Agents. Journal of Cancer Therapy, 6, 40-44. http://dx.doi.org/10.4236/jct.2015.61006 
mors and create arguably the greatest challenge in oncology [1]-[4]. These tumors may constitute up to 85\% of gliomas found within the pediatric brainstem [1]-[4]. However, DIPG in young adult patients is rare, with the estimated number of cases a year in the United States to be below 20 [5] [6].

The brainstem controls extremely important bodily functions, including control of heart rhythm and breathing. Due to the strategic location of brainstem gliomas, surgery and even biopsy may endanger a patient's life. Since biopsy is difficult, dangerous and sometimes misleading, diagnosis based on magnetic resonance imaging (MRI) remains the diagnostic standard [3] [7] [8].

All recent clinical studies for brainstem gliomas in the United States define DIPGs as tumors involving more than $50 \%$ of the pons or tumors which have anaplastic or glioblastoma multiforme (GBM) histology occupying less than $50 \%$ of pons [8]. The medical literature does not offer a different classification for adult DIPG [5] [6].

Sodium phenylbutyrate (PB) is a drug approved by USA Food and Drug Administration (FDA) for the treatment of episodic hyperammonemia in children with inborn errors of urea synthesis [9]. PB is a histone deacetylase inhibitor (HDAC) and has been studied in numerous other disorders, including brain tumors [10]. During its metabolism, the molecule is broken down in the body to phenylacetate (PN), which is then conjugated with a glutamine forming phenylacetylglutamine (PG) [9] [10]. The three compounds (i.e. PB, PG, and PN) all contribute to biological activity in the body. PG and PN are also ingredients of antineoplastons (ANP) A10 and AS2-1, which is currently undergoing development for the treatment of various brain tumors by Burzynski Research Institute (BRI) [11]-[13]. Preclinical data indicated that PG and PN affect over 100 genetic targets involved in the development of high-grade glioma [14]. Several recently completed clinical studies, revealed a number of complete and partial responses in DIPG, including a long-term survival exceeding 26 years [15]-[22]. Since there is no established standard-of-care for recurrent DIPG (RDIPG), a treatment plan for such patients is proposed, which includes PB and a combination of targeted agents [20]. This article reports successful therapy for RDIPG according to such strategy.

\section{Clinical Presentation}

In October 2012, a 33-year-old Caucasian male developed numbness on the left side of his body. A computerized tomography (CT) scan of the head revealed an ill-defined patchy area of decreased attenuation in the pons. An MRI of the head shortly thereafter had shown a complex cystic rim-enhancing tumor located centrally within the pons surrounded by vasogenic edema and exerting mass effect on the $4^{\text {th }}$ ventricle consistent with DIPG. From October 22 to December 4, 2012, the patient received standard radiation therapy to the brainstem for a total of 30 treatments. It was felt that neither surgery nor chemotherapy was beneficial for this type of tumor. The follow-up MRI of the head of January 5, 2013 revealed an increase in the size of the lesion since October 13, 2012.

At the beginning of 2013, the patient presented to the BC, complaining of decreased superficial sensation on the left side of his body. He had a balance disturbance and his left ear was more sensitive to sound. Physical examination was significant for facial asymmetry with signs of left facial nerve paralysis, slurred speech, left flaccid hemiplegia, unsteady gate, and poor coordination. Deep tendon reflexes were reduced on the left side and Babinski was positive on the left. Karnofsky Performance Status (KPS) was 60. MRI of the head, with and without contrast, on January 23, 2013 revealed brainstem contrast-enhancing lesion located in the pons measuring $2.8 \times 2.4 \mathrm{~cm}$. Laboratory tests were significant for hypercholesterolemia and the elevation of the extracellular domain of vascular endothelial growth factor (VEGF).

On January 24, 2013, the patient initiated treatment with oral PB, receiving a dose of 3 g six times daily. On January 25, 2013, he began intravenous (IV) infusion of bevacizumab (BVZ) and was advised to increase the dose to $10 \mathrm{mg} / \mathrm{kg}$ (870 mg daily) every two weeks. Within a week, he initiated orally administered pazopanib $200 \mathrm{mg}$ daily, everolimus $10 \mathrm{mg}$ every other day and erlotinib $150 \mathrm{mg}$ daily. In total, he received approximately 1.5 years of treatment with PB, pazopanib, erlotinib, and BVZ, though everolimus was discontinued after 5 months.

The patient accomplished reduction and finally, elimination of his neurological symptoms. He had temporary improvement of his left hemiplegia after 5 days and significant improvement after 5 weeks of treatment. He was symptom-free after 9 months. MRI of the head on March 11, 2013, revealed approximately 60\% decrease of the contrast-enhancing tumor. The follow-up MRI of June 20, 2013 showed an 82\% decrease of the tumor (Figure 1). On May 28, 2014, the tumor began to increase, but was still 53\% smaller than baseline. The patient decided 


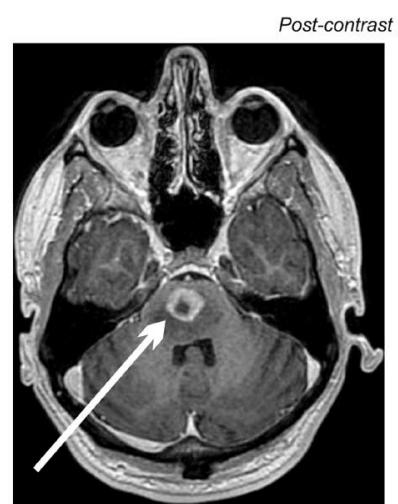

Pre-baseline MRI - Oct. 13, 2012

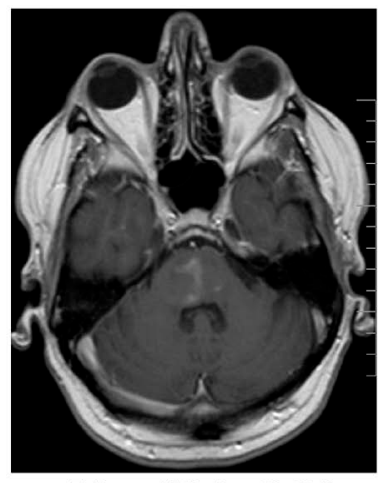

Follow-up MRI - June 20, 2013

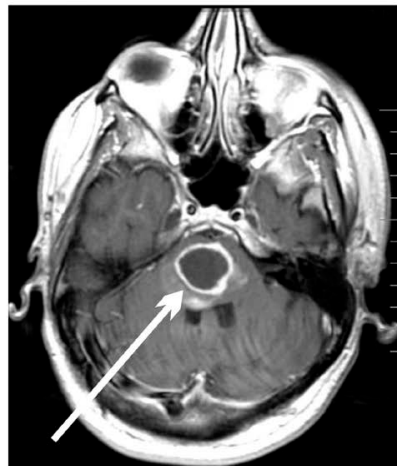

Baseline MRI - Jan. 23, 2013

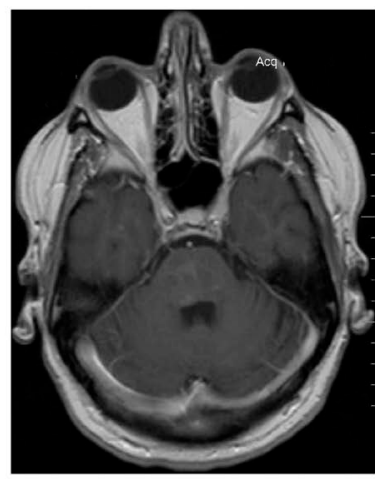

Follow-up MRI - Aug. 15, 2013

Figure 1. Pre-baseline (before radiation), baseline (after radiation), and follow-up (on treatment at Burzynski Clinic) MRIs of the head, which illustrate a decrease in tumor size.

to discontinue the medications on July 25, 2014. A month later, he opted to start treatment with temozolomide (TMZ) for one month. Unfortunately, his disease progressed and he passed away on September 28, 2014.

Additional drugs included dexamethasone, which was taken pretreatment (4 mg daily). Initially, the dexamethasone dose was increased to $8 \mathrm{mg}$ daily (15 days), then reduced to daily doses of $6 \mathrm{mg}$ (83 days), to $5 \mathrm{mg}$ (60 days), then to $2 \mathrm{mg}$ ( 2 days) and ultimately discontinued. He was also receiving doxycycline $100 \mathrm{mg}$ b.i.d. (30 days) and clindamycin gel b.i.d. (30 days) to reduce a skin rash and lansoprazole $20 \mathrm{mg}$ daily (30 days).

Toxicity was graded according to Common Terminology Criteria for Adverse Events v3.0 (CTCAE).

The treatment was associated with minimal adverse events that included Grade 1 and Grade 2 skin rash and Grade 1 elevation of alanine aminotransferase (ALT) and aspartate aminotransferase (AST). The most unpleasant adverse event was a skin rash related to erlotinib. Elevation of transaminases was related to everolimus, which was discontinued at that time. Overall survival from diagnosis was one year and eleven months, from treatment start was one year and nine months, and progression-free survival was one year and five months.

\section{Discussion}

Standard-of-care for newly-diagnosed DIPG is radiation therapy, which was administered in the described case. Unfortunately, there is no established standard therapy for RDIPG [2]-[4]. Single or multiagent chemotherapy and targeted therapy regimens have not demonstrated efficacy in such cases and there were no phase II clinical trials in RDIPG conducted in adult patients [5] [6]. Based on our successful treatment of recurrent GBM (RGBM), we used a similar strategy for patients with RDIPG, despite reservations in using such a treatment plan, since the "genomic signature" of DIPG is different than GBM and is characterized by mutations in the histone H3.3 (H3F3A) [20] [23]. Medications proposed for treatment regulate VEGF, epidermal growth factor (EGFR), and mammalian target of rapamycin (mTOR) pathways as well as interrupt the signal transmission through AKT and RAS pathways and affect cyclin-dependent kinases (CDK), and cyclins. The rationale for the combination 
of targeted agents including pazopanib, everolimus, erlotinib, BVZ, dasatinib (recommended, but not started), and PB was previously explained in detail [14] [20]. A dose reduction of 50\% to $75 \%$ for PB, pazopanib, and everolimus was effected to diminish the possibility of additive toxicity of the regimen. The treatment resulted in a rapid decrease in the tumor size and the onset of a partial response after seven weeks. The maximum reduction in the tumor size was in excess of $80 \%$. There were some noted deficiencies in the execution of the treatment plan. Everolimus was discontinued by the patient himself after five months due to elevation of transaminases and dasatinib was not added to the treatment (based on financial concerns). It is postulated that noncompliance may have resulted in progression of the disease after one year and five months. After the patient opted to discontinue his targeted treatment regimen (for financial reasons) and take TMZ instead, the patient had a rapid progression of his disease and died within two months thereafter. Factors contributing to noncompliance were high cost of medications and a lack of insurance coverage.

It should be noted that the baseline evaluation was performed after seven weeks from the completion of RT, raising the possibility of pseudoprogression. To eliminate this possibility, it is appropriate to wait an additional five weeks before starting treatment, however, the patient's clinical deterioration prompted us to begin his therapy sooner.

\section{Conclusions}

This case report suggests that it is possible to obtain objective response in the treatment of RDIPG in a young adult patient using a combination of gene targeted agents. The patient achieved close to a two-year survival, which appeared substantially longer than the median survival of eleven months for this age group [5] [6]. With appropriate dose reductions, a targeted treatment regimen can be safely administered. However, caution should be exercised since formal clinical trials were not conducted.

Results of using PB in combination with targeted agents pazopanib, everolimus, erlotinib/dasatinib, and BVZ need to be confirmed in future clinical trials.

\section{Conflicts of Interest}

All authors are employed by Burzynski Clinic. Dr. Stanislaw R. Burzynski is President at the Burzynski Clinic. Dr. Gregory S. Burzynski is Vice-President of Burzynski Clinic and Dr. Sheldon Brookman is Director of Pharmaceutical Development of Burzynski Clinic.

\section{Acknowledgements}

The authors express their appreciation to the additional physicians involved in the care of the patients: Drs. Zanhua Yi, Alejandro Marquis, and Mohammad Khan. Preparation of the manuscript was provided by Carolyn Powers, Adam Golunski, and Jennifer Pineda.

\section{References}

[1] Ostrom, Q.T., Gittleman, H., Liao, P., Rouse, C., Chen, Y., et al. (2014) CBTRUS Statistical Report: Primary Brain and Central Nervous System Tumors Diagnosed in the United States in 2007-2011. Neuro-Oncology, 16, iv1-iv63. http://dx.doi.org/10.1093/neuonc/nou223

[2] Donaldson, S.S., Laningham, F. and Fisher, P.G. (2006) Advances toward an Understanding of Brainstem Gliomas. Journal of Clinical Oncology, 24, 1266-1272. http://dx.doi.org/10.1200/JCO.2005.04.6599

[3] Hargrave, D., Bartels, U. and Bouffet, E. (2006) Diffuse Brainstem Glioma in Children: Critical Review of Clinical Trials. Lancet, 7, 241-248. http://dx.doi.org/10.1016/S1470-2045(06)70615-5

[4] Burzynski, S.R. (2007) Recent Clinical Trials in Diffuse Intrinsic Brainstem Glioma. Cancer Therapy, 5, 379-390.

[5] Guillamo, J.S., Monjour, A., Taillandier, L., Devaux, B., Varlet, P., et al. (2001) Brainstem Gliomas in Adults: Prognostic Factors and Classification. Brain, 124, 2528-2539. http://dx.doi.org/10.1093/brain/124.12.2528

[6] Kesari, S., Kim, R.S., Markos, V., Drappatz, J., Wen, P.Y. and Pruitt, A.A. (2008) Prognostic Factors in Adult Brainstem Gliomas: A Multicenter, Retrospective Analysis of 101 Cases. Journal of Neuro-Oncology, 88, 175-183. http://dx.doi.org/10.1007/s11060-008-9545-1

[7] Barkovich, A.J., Krischer, J., Kun, L.E., Packer, R., Zimmerman, R.A., et al. (1990) Brain Stem Gliomas: A Classification System Based on Magnetic Resonance Imaging. Pediatric Neurosurgery, 16, 73-83. 
http://dx.doi.org/10.1159/000120511

[8] Albright, A.L., Packer, R.J., Zimmerman, R., Rorke, L.B., Boyett, J. and Hammond, G.D. (2013) Magnetic Resonance Scans Should Replace Biopsies for the Diagnosis of Diffuse Brain Stem Gliomas: A Report from the Children's Cancer Group. Neuro-Oncology, 15, 1026-1030.

[9] Brusilow, S.W., Danney, M., Waber, L.J., Batshaw, M., Burton, B., et al. (1984) Treatment of Episodic Hyperammonemia in Children with Inborn Errors of Urea Synthesis. New England Journal of Medicine, 310, 1630-1634. http://dx.doi.org/10.1056/NEJM198406213102503

[10] Iannitti, T. and Palmieri, B. (2011) Clinical and Experimental Applications of Sodium Phenylbutyrate. Drugs in R\&D, 11, 227-249. http://dx.doi.org/10.2165/11591280-000000000-00000

[11] Burzynski, S.R. (1976) Antineoplastons: Biochemical Defense against Cancer. Physiological Chemistry and Physics, 8, 275-279.

[12] Burzynski, S.R. (1986) Antineoplastons: History of the Research (I). Drugs under Experimental and Clinical Research, 12, 1-9.

[13] Burzynski, S.R. (2004) The Present State of Antineoplaston Research (1). Integrative Cancer Therapies, 3, 47-58. http://dx.doi.org/10.1177/1534735403261964

[14] Burzynski, S.R. and Patil, S. (2014) The Effect of Antineoplastons A10 and AS2-1 and Metabolites of Sodium Phenylbutyrate on Gene Expression in Glioblastoma Multiforme. Journal of Cancer Therapy, 5, 929-945. http://dx.doi.org/10.4236/jct.2014.510099

[15] Burzynski, S.R. (2006) Treatments for Astrocytic Tumors in Children: Current and Emerging Strategies. Pediatric Drugs, 8, 167-168. http://dx.doi.org/10.2165/00148581-200608030-00003

[16] Burzynski, S.R., Janicki, T.J., Burzynski, G.S. and Marszalek, A. (2014) The Response and Survival of Children with Recurrent Diffuse Intrinsic Pontine Glioma Based on Phase II Study of Antineoplastons A10 and AS2-1 in Patients with Brainstem Glioma. Child's Nervous System. http://dx.doi.org/10.1007/s00381-014-2401-z

[17] Burzynski, S.R., Janicki, T.J., Burzynski, G.S. and Marszalek, A. (2014) A Phase II Study of Antineoplastons A10 and AS2-1 in Children with High-Grade Glioma. Final Report and Review of Recent Trials. Journal of Cancer Therapy, 5 , 565-577. http://dx.doi.org/10.4236/jct.2014.56065

[18] Burzynski, S.R., Janicki, T.J. and Burzynski, G.S. (2014) A Phase II Study of Antineoplastons A10 and AS2-1 in Adult Patients with Recurrent Glioblastoma Multiforme. Final Report (Protocol BT-21). Journal of Cancer Therapy, 5, 946-956. http://dx.doi.org/10.4236/jct.2014.510100

[19] Burzynski, S.R., Janicki, T.J., Burzynski, G.S. and Marszalek, A. (2014) A Phase II Study of Antineoplastons A10 and AS2-1 in Children with Recurrent, Refractory or Progressive Primary Brain Tumors. Final Report (Protocol BT-22). Journal of Cancer Therapy, 5, 977-988. http://dx.doi.org/10.4236/jct.2014.510102

[20] Burzynski, S.R., Burzynski, G.S. and Janicki, T.J. (2014) Recurrent Glioblastoma Multiforme—A Strategy for LongTerm Survival. Journal of Cancer Therapy, 5, 957-976. http://dx.doi.org/10.4236/jct.2014.510101

[21] Burzynski, S.R., Kubove, E. and Burzynski, B. (1992) Phase II Clinical Trials of Antineoplastons A10 and AS2-1 Infusions in Astrocytoma. In: Adam, D., Ed., Recent Advances in Chemotherapy, Futuramed Publishers, Munich.

[22] Burzynski, S.R., Janicki, T.J., Burzynski, G.S. and Marszalek, A. (2013) Long-Term Survival (>13 years) in a Child with Recurrent Diffuse Pontine Gliosarcoma: A Case Report. Journal of Pediatric Hematology Oncology.

[23] Khuong-Quang, D.A., Buczkowicz, P., Rakopoulos, P., Liu, X.Y., Fontebasso, A.M., et al. (2012) K27M Mutation in Histone H3.3 Defines Clinically and Biologically Distinct Subgroups of Pediatric Diffuse Intrinsic Pontine Gliomas. Acta Neuropathologica, 124, 439-447. http://dx.doi.org/10.1007/s00401-012-0998-0 
Scientific Research Publishing (SCIRP) is one of the largest Open Access journal publishers. It is currently publishing more than 200 open access, online, peer-reviewed journals covering a wide range of academic disciplines. SCIRP serves the worldwide academic communities and contributes to the progress and application of science with its publication.

Other selected journals from SCIRP are listed as below. Submit your manuscript to us via either submit@scirp.org or Online Submission Portal.
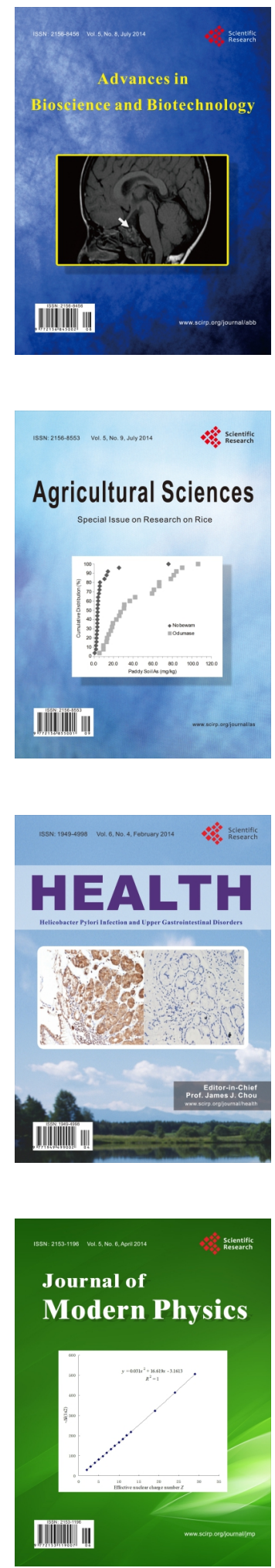
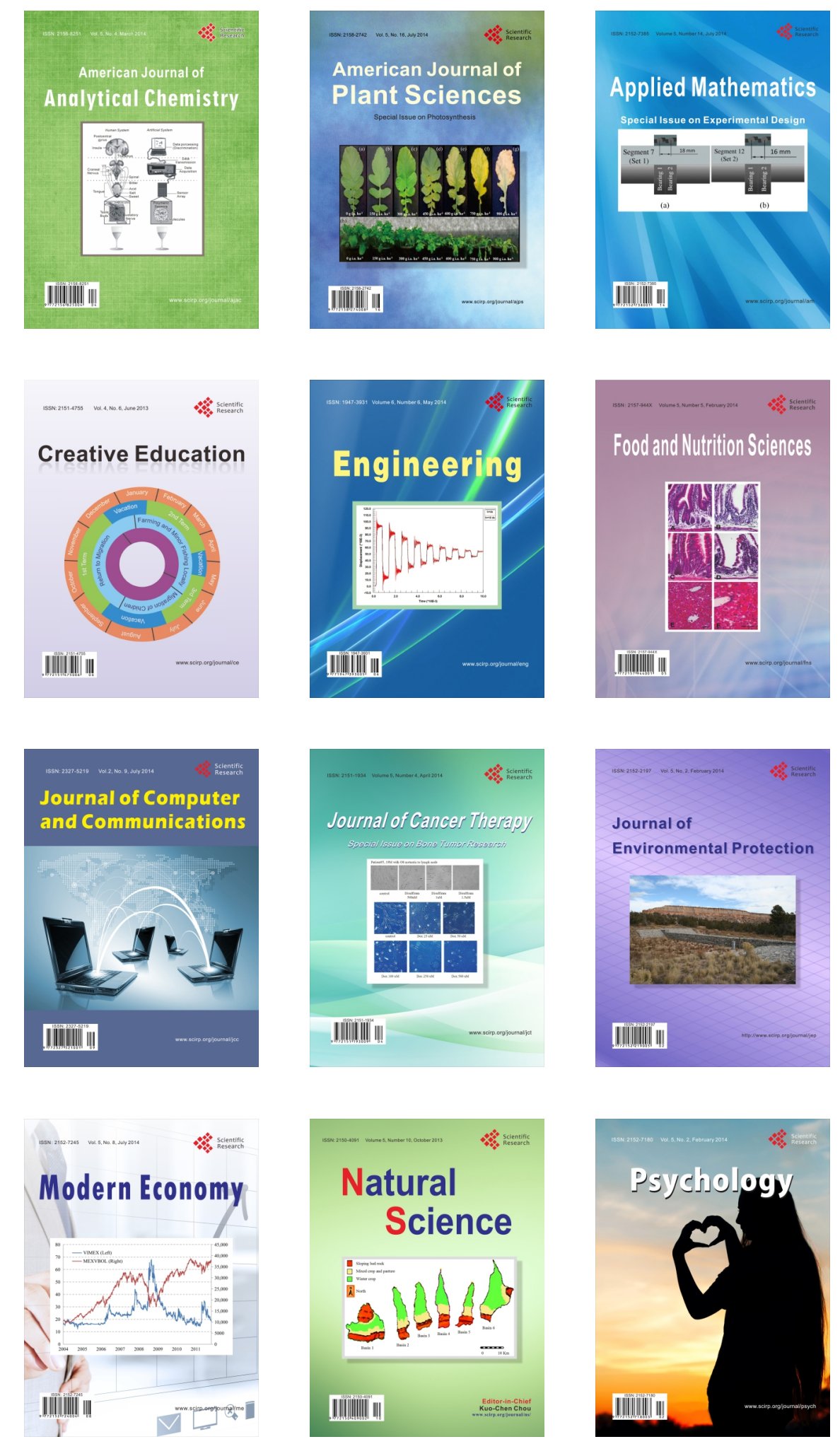